\title{
Stimulation of Gut Immune System by Early Administration of Probiotic Strains in Dicentrarchus labrax and Sparus aurata
}

\author{
Luigi Abelli, ${ }^{a}$ Elisa Randelli, ${ }^{b}$ Oliana Carnevali, ${ }^{c}$ \\ and Simona Picchietti ${ }^{b}$ \\ ${ }^{a}$ Department of Biology and Evolution, Comparative Anatomy Section, \\ Ferrara University, Ferrara, Italy \\ ${ }^{b}$ Department of Environmental Sciences, Tuscia University, Viterbo, Italy \\ ${ }^{c}$ Department of Marine Sciences, Marche Polytechnic University, Ancona, Italy
}

\begin{abstract}
Early feeding (started during gut metamorphosis and establishment of the hypothalamic-pituitary-interrenal axis) with probiotic-supplemented diets, besides modifying the intestinal microflora, evoked profound effects on the physiology of fish larvae. Using rotifers and Artemia as living vectors, the autochthonous bacterium Lactobacillus delbrueckii delbrueckii or a multispecies probiotic formulation (autochthonous Lactobacillus fructivorans + Lactobacillus plantarum from human feces) were orally administered to sea bass and gilthead sea bream larvae, respectively. The treatments enhanced larval rearing (significantly increased body weight, decreased cortisol levels, and improved stress response compared with controls) and the immune system. In sea bass, the probiotic raised intestinal $T$ cells, in keeping with increased total body $T c R-\beta$ transcripts, and increased acidophilic granulocytes concomitant to lower transcription of pro-inflammatory genes $(I L-1-\beta, T G F-\beta, I L-10, C o x-2)$. In sea bream, the multispecies probiotic formulation raised intestinal immunoglobulin $\left(\mathrm{Ig}^{+}\right)$cells and acidophilic granulocytes mainly belonging to the $\mathrm{MAb} \mathrm{G} 7^{+}$phagocytic population. These results point to stimulatory actions of probiotics on the gut immune system that are devoid of harmful effects and that correlate with improvement of fry survival.
\end{abstract}

Key words: Lactobacillus; probiotic; larval immunity; teleost fish

\section{Introduction}

The use of probiotics or beneficial bacteria in animal nutrition is well documented and has recently begun to be applied in fish aquaculture as an alternative to antibiotics.

The probiotic strain Lactobacillus delbrueckii ssp. delbrueckii (AS13B), isolated from intestinal microflora of adult sea bass, ${ }^{1}$ was administered to Dicentrarchus labrax larvae and postlarvae, using Brachionus plicatilis and Artemia salina as vectors, to investigate beneficial immunomodula-

Address for correspondence: Professor Luigi Abelli, Department of Biology and Evolution, Section of Comparative Anatomy, University of Ferrara, Via Borsari 46, 44100 Ferrara, Italy. Fax: 0532-455715. abl@unife.it tory properties and the effects on development and differentiation of gut-associated lymphoid tissue. We previously verified in fish from the same experiment that the bacterial strain produced desirable effects during development; this was demonstrated by a significant increase of larval body weight and decrease of cortisol levels. ${ }^{1}$

Likewise, the two bacterial strains Lactobacillus fructivorans (AS17B), isolated from adult sea bream intestinal microflora, ${ }^{2}$ and Lactobacillus plantarum (906), isolated from human feces, ${ }^{3}$ were administered to Sparus aurata larvae and postlarvae using the living vectors as above. These bacterial strains positively affected sea bream development, which was 